\title{
Monitoramento e avaliação na atenção básica no Brasil: a experiência recente e desafios para a sua consolidação
}

\author{
Monitoring and evaluation in primary care in Brazil: recent \\ experience and challenges for its consolidation
}

Allan Nuno Sousa ${ }^{1}$

DOI: $10.1590 / 0103-110420185119$

\section{Introdução}

O objetivo do presente ensaio é apresentar um apanhado dos principais avanços na agenda de monitoramento e avaliação da atenção básica, frutos da iniciativa ou investimento direto do Ministério da Saúde ao longo das últimas duas décadas, apontando para alguns dos mais importantes desafios para a implementação da agenda de institucionalização da avaliação no contexto da Política Nacional de Atenção Básica.

Inicialmente, trataremos da temática do monitoramento e avaliação a partir de abordagens e conceitos construídos no campo da análise de políticas públicas e de avaliação em saúde, com o objetivo de delinear os pressupostos dos quais partimos para a interpretação dos mecanismos que construíram e consolidaram uma agenda de fortalecimento das práticas avaliativas no âmbito da atenção básica no Brasil. Como procuraremos argumentar, independentemente dos resultados encontrados a partir do esforço de empreender processos avaliativos, as condições políticas, econômicas e sociais constituem elementos suficientemente fortes para o constrangimento das ações dos atores e para a definição do leque de possíveis problemas e ações a serem consideradas por eles.

Na sequência, procuraremos descrever os principais eventos, iniciativas e ações que foram dando contorno à história da política de monitoramento e avaliação da atenção básica que veio sendo construída desde o início dos anos 2000 até os dias atuais. Tentaremos lançar luz sobre como se inseriu, quais foram os fatores que contribuíram para o fortalecimento, os avanços e quais os principais desafios para a consolidação da avaliação no âmbito da atenção básica. Para isso, visitaremos a literatura recente sobre avaliação em saúde e desenvolveremos uma análise documental, considerando as principais publicações oficiais e 'literatura cinzenta' que envolvem o tema do monitoramento e avaliação na atenção básica.

1 Ministério da Saúde Brasília (DF), Brasil. Orcid: https://orcid. org/0000-0002-86891347

alnunos@gmail.com

\section{Monitoramento e avaliação em saúde}

O campo da avaliação em saúde tem se expandido de maneira progressiva ao longo das últimas décadas, tanto como disciplina teórico-metodológica, quanto como um campo de práticas, 
racionalmente orientadas, de sujeitos interessados em transformar uma dada situação em saúde na direção de outra mais desejável, considerando um conjunto de princípios e valores ético-políticos.

$\mathrm{Na}$ literatura especializada, há um vasto e, por vezes, difuso espectro de conceitos que delimitam aquilo que deve ser entendido como monitoramento e avaliação em saúde ${ }^{\mathbf{1 , 2}}$. Contudo, alguns aspectos parecem corresponder a um certo consenso a respeito da ideia geral do que venha a ser monitoramento e avaliação. Ao passo que o monitoramento representa o rol de esforços para o acompanhamento sistemático a respeito de algum dos aspectos de uma determinada intervenção, por meio de indicadores mensurados a partir da coleta continua de dados, a avaliação corresponde ao exercício de mensurar, compreender e julgar os efeitos de uma determinada intervenção, de maneira a subsidiar as escolhas da comunidade política no processo de tomada de decisão, seja ele na etapa de formulação ou implementação da intervenção avaliada. A partir dessa ideia, o monitoramento e a avaliação apresentam fortes elementos de complementariedade, na medida em que as atividades relacionadas com o processo de monitoramento podem contribuir, sobremaneira, para a produção de informações que ancorem a realização de processos avaliativos.

A literatura que aborda o desenvolvimento das políticas públicas tem, cada vez mais, afastado-se de modelos analíticos que pressupõem a avaliação como uma etapa de um processo linear, no qual tem a responsabilidade de indicar, ao final da implementação de uma dada política, o sucesso ou fracasso, seja do seu todo ou de partes que a compõe. Torna-se cada vez mais patente que o emprego da avaliação deve ocorrer desde a montagem da agenda (entrada de problemas no rol de preocupação de governos), passando pelo processo de formulação das alternativas viáveis que podem contribuir para a resolução dos problemas identificados, pelo processo de tomada de decisão das alternativas apresentadas e pela implementação das políticas eleitas pelos agentes públicos. Em cada uma dessas etapas, conteúdos produzidos por avaliações devem ser mobilizados para subsidiar melhores escolhas por parte dos atores envolvidos, seja qual for o momento em que se encontra o desenvolvimento da política pública ${ }^{3}$.

Para Patton ${ }^{4}$, a avaliação possui três objetivos primários, quais sejam: (a) julgar os resultados produzidos por uma ação, programa ou política; (b) contribuir para o seu desenvolvimento e (c) apoiar a construção de conhecimento a respeito do fenômeno avaliado. No entanto, para além desses objetivos, como chama a atenção Faria 5 , é importante destacar o caráter político-simbólico das iniciativas avaliativas. Para o autor, as avaliações, além de suportar as ações de planejamento e identificação de problemas que precisam ser enfrentados pelos agentes públicos, possuem um forte caráter político. Elas preenchem importantes lacunas de informação a respeito dos resultados alcançados pela ação do poder público, sobre a alocação (ou realocação) dos recursos oriundos dos tributos e conferem legitimidade a ação política e burocrática. Nesse sentido, para além do uso instrumental na qual as avaliações tradicionalmente são mobilizadas, é importante destacar seu elemento democratizador, na medida em que se apresenta como importante dispositivo de amplificação da responsividade do Estado ante as demandas da sociedade.

Outro aspecto de extrema relevância diz respeito à contribuição da avaliação para a promoção da mudança nos rumos das políticas. A tendência da literatura no campo da análise de políticas públicas é o de entender as mudanças como meras adaptações ou ajustes para alterar circunstâncias constrangidas pela reprodução contínua de políticas existentes. A ênfase na estabilidade institucional está associada à ideia de que, por presunção, existem constrangimentos 
suficientemente fortes que garantem a continuidade ${ }^{3}$. Contudo, entendemos que a avaliação das políticas pode contribuir para a alimentação de certa dinamicidade que confronta o instituído e que apresenta alternativas, ajustadas a novas situações, compreensões da realidade e demandas, ainda que existam fortes elementos que reforçam a estabilidade das políticas.

O esforço avaliativo pode envolver, em um extremo, a realização de pesquisas de natureza estritamente acadêmica, profundamente preocupadas com aspectos teóricos, conceituais e metodológicos que confiram validade científica aos achados dos estudos; e no outro, o emprego de um conjunto variado de técnicas e procedimentos que suportem a prática cotidiana de gestores e trabalhadores, de maneira mais perene e fluida, menos preocupadas com os melindres da robustez metodológica, sem, contudo, descuidar da importância do método e de sua implicação para o reconhecimento do valor dos conteúdos produzidos.

Na experiência recente brasileira, nesse amplo espectro de possibilidades metodológicas, um conjunto elevado de iniciativas emergem de diversas frentes, fontes e perspectivas epistemológicas, indicando uma substancial elevação da relevância das práticas avaliativas no campo da saúde e, em particular nas ações que abrangem os esforços de qualificação da atenção básica no País. No campo orientado para a racionalização dos processos de gestão, o monitoramento e a avaliação ganham maior espaço, principalmente a partir dos anos 1990, na esteira das ações empreendidas pelos governos para promover um processo de reforma do Estado, associado a exigências de organismos internacionais que, ao destinar recursos para a implementação de políticas e programas, determinavam a introdução de planos e ações que pudessem garantir o acompanhamento dos programas ao longo de sua implementação e a avaliação de seus resultados.

\section{Entrada na agenda: o fortalecimento das ações de monitoramento e avaliação na atenção básica no âmbito da gestão federal (2001- 2010)}

O Sistema Único de Saúde (SUS) emergiu em um contexto histórico no qual a extensão dos direitos sociais se apresenta como elemento central no processo de produção do marco institucional após o período autoritário vivenciado pelo País na segunda metade do século XX. A luta pela ampliação dos direitos sociais, que garantissem o aumento do bem-estar e da capacidade produtiva e de consumo da sociedade, passou a integrar o escopo de condições necessárias para a construção de uma sociedade democrática no Brasil. Nesse contexto, importantes estratégias foram sendo gestadas do âmbito do SUS, com o intuito de promover seus princípios e diretrizes, como a universalidade e a integralidade do cuidado. Dentre elas, a atenção básica se destaca por sua capilaridade e capacidade de produzir um deslocamento de uma dada ênfase do cuidado em saúde, quase que exclusivamente centrada nos serviços, para uma perspectiva que considera que as condições de saúde dos indivíduos estão intimamente relacionadas com as dimensões sociais, políticas e econômicas em que estão inseridos ${ }^{6}$.

A atenção básica no Brasil foi se constituindo de maneira heterogênea, estando sistematicamente associada à capacidade fiscal dos municípios, uma vez que não havia, até a construção do SUS, uma política nacional estruturada para atender às demandas locais para o desenvolvimento da atenção básica, e associada a preferências ideológicas dos governantes municipais. Foi apenas em 1996 que o Governo Federal instituiu um mecanismo regular e universal de transferência de recursos aos municípios, condicionados ao porte populacional e à organização e prestação de serviços em atenção básica, o Piso de Atenção Básica (PAB). 
A partir de então, o tema do monitoramento e avaliação passou, ainda que timidamente, a ser incorporado no âmbito da gestão federal, principalmente em função das exigências administrativas de controle dos repasses para a implantação da atenção básica no País. Em 1998, o Ministério da Saúde publicou um documento intitulado 'manual para a organização da atenção básica', no qual determina um conjunto de regramentos para o funcionamento da atenção básica; e o tema do monitoramento e avalição começa a ganhar maior relevo nas estruturas político-gerenciais de funcionamento dos projetos e programas de atenção básica que passaram a ser mais bem planejados e acompanhados pelos gestores do SUS7.

Tal manual, em seu capítulo que versa sobre as responsabilidades da gestão, associa a avaliação a mecanismos de controle e prevê a obrigatoriedade dos gestores de elaborar rotinas, normas e fluxos de controle e avaliação. Esse traço da publicação reforça sua concepção de avaliação como instrumento gerencial de controle das atividades dos municípios, por parte do governo central. Contudo, a mesma publicação já sinaliza em direção a uma percepção mais ampliada da função avaliativa no âmbito geral de funcionamento da atenção básica nos munícipios, na medida em que prevê a importância da participação dos trabalhadores das equipes e dos conselhos municipais de saúde no processo de planejamento, desenvolvimento e avaliação das ações dos serviços de atenção básica.

No entanto, foi somente a partir da instituição na estrutura formal do Ministério da Saúde, de uma unidade dedicada às atividades de monitoramento e avaliação da política de atenção básica e dos projetos prioritários de governo, a Coordenação de Acompanhamento e Avaliação do Departamento de Atenção Básica, que o tema passou a ocupar um papel de destaque na gestão federal. Foi a partir daí que se desenvolveu um conjunto de projetos e ações que culminaram na publicação de uma
Política de Monitoramento e Avaliação da Atenção Básica no ano de $2006^{8}$.

Ainda em 2002, o Ministério da Saúde empreendeu o maior estudo feito até então para avaliar a situação da atenção básica no País?. Tratou-se de um estudo censitário, no qual foram avaliadas 13.501 equipes de saúde da família em cerca de $70 \%$ dos municípios brasileiros. Os resultados desse estudo passaram a representar, até então, o maior retrato já construído a respeito das condições em que se encontravam as unidades básicas de saúde e equipes de saúde da família. Foram levantadas questões relacionadas com o funcionamento geral das unidades, ao acesso, infraestrutura, gestão do trabalho, processo de trabalho das equipes e a sua relação com os demais pontos da rede.

No primeiro semestre do mesmo ano, a partir de um acordo de empréstimo firmado entre o governo brasileiro e o Banco Mundial, o Ministério da Saúde instituiu o Projeto de Expansão e Consolidação do Saúde da Família (Proesf), cujo objetivo principal era estimular a conversão do modelo de atenção básica e expandir a cobertura populacional da saúde da família nas grandes cidades brasileiras (municípios acima de 100 mil habitantes), que naquele período representava o maior desafio para o aumento das coberturas.

O Proesf estava previsto para ser executado em três fases, sendo que a primeira contou com um total de US\$ 68 milhões, iniciada em 2002 e concluída em meados de 2007. Entre seus componentes, havia aquele destinado a investimentos na gestão federal, ao apoio à execução do projeto nos municípios participantes e às iniciativas de monitoramento e avaliação.

$\mathrm{Na}$ esteira dos investimentos viabilizados a partir do Proesf, a coordenação de acompanhamento e avaliação desenvolveu um plano estratégico prevendo uma série de medidas que visavam contribuir para o processo de institucionalização da avaliação na atenção básica. A ideia de institucionalização da avaliação está intimamente relacionada com a agenda de modernização da capacidade de 
gestão e gestão do cuidado nos serviços de saúde, pressupondo a indução e o estímulo a incorporação cotidiana de práticas avaliativas no interior das organizações 8,10,11. Entre os eixos estruturadores do plano estratégico para a institucionalização da avaliação no âmbito da atenção básica, destacaram-se o desenvolvimento da capacidade avaliativa dos atores, a cooperação técnica entre a gestão e a academia, a indução de estudos e pesquisas e a produção de informação e comunicação.

Entre as principais ações relacionadas com a agenda de institucionalização da avaliação, ganharam maior visibilidade a realização dos estudos de linha de base do Proesf, o fomento de estudos e pesquisas avaliativas, a construção e implantação de uma ferramenta para a autoavaliação das equipes e o investimento em ações para o fortalecimento da capacidade técnica das Secretarias de Estado da Saúde em monitoramento e avaliação da atenção básica.

Os estudos de linha de base do Proesf possuíam como objetivo principal o estabelecimento de uma linha de base para uma posterior avaliação de impacto do projeto ao término de sua terceira fase. Ele iniciou em 2004 e contou com a participação de oito instituições de ensino e pesquisa, que se encarregaram de avaliar a organização da atenção básica, incluindo práticas de gestão e de oferta de serviços, e o cuidado aos usuários, considerando, entre outras coisas, o acolhimento, vínculo e a qualidade do cuidado ${ }^{\mathbf{1 2}}$.

Relacionado ao componente de indução de estudos e pesquisas da política de monitoramento e avaliação da atenção básica, em 2005, por meio de editais de pesquisa, a partir de uma parceria do Departamento de Atenção Básica com o Conselho Nacional de Desenvolvimento Científico e Tecnológico (CNPq), foram financiados 84 projetos de estudos e pesquisas, com largo espectro de objetos de análise que envolveram cinco grandes eixos, (1) avaliação da gestão e reorganização da atenção básica em municípios acima de 100 mil habitantes, (2) complexo produtivo em saúde e financiamento da atenção básica, (3) institucionalização da avaliação na atenção básica no âmbito do SUS, (4) desenvolvimento e validação de metodologias de análise e avaliação da atenção básica e (5) processo de trabalho em saúde ${ }^{13}$.

No componente destinado a investir na capacidade avaliativa dos atores, duas importantes iniciativas foram implementadas: (a) o projeto de fortalecimento da capacidade técnica em monitoramento e avaliação das Secretarias de Estado da Saúde, cujo principal objetivo era apoiar o processo de formação dos gestores estaduais da atenção básica para o desenvolvimento do monitoramento e avalição ${ }^{\mathbf{1 4}}$; (b) e a ferramenta de Autoavaliação para a Melhoria da Qualidade (AMQ), que tinha como principal objetivo oferecer um método que auxiliasse gestores e trabalhadores nas suas ações de planejamento, por meio da identificação dos avanços e das necessidades de qualificação das suas práticas, por meio do auto reconhecimento e da pactuação de compromissos coletivos ${ }^{15}$.

Em 2006, o Ministério da Saúde publicou portaria que passou a ser conhecida como Política Nacional da Atenção Básica, documento no qual evidencia de maneira mais clara, em contraste com a portaria que normatizava o funcionamento da atenção básica anterior, a importância do monitoramento e avaliação para o desenvolvimento da atenção básica no País. Entre os fundamentos da política, encontram-se demarcados no documento a realização da avaliação e o acompanhamento sistemático dos resultados alcançados, como parte do processo de planejamento e programação, e entre as atribuições de gestores, nos três níveis de gestão, está a definição de estratégias de articulação com os serviços de saúde com vistas à institucionalização da avaliação da atenção básica ${ }^{16}$. Essa explicitação revela a forte prioridade da gestão federal no campo do fortalecimento da avaliação em saúde, particularmente na atenção básica, e contribuiu para o desencadeamento de um conjunto de 
ações que, ao longo dos próximos anos colocaria a prática avaliativa no centro do debate da Política Nacional de Atenção Básica.

No primeiro semestre de 2008, o Ministério da Saúde iniciou a segunda fase do Proesf, garantindo em seu escopo geral e em componentes específicos a continuidade de ações relacionadas diretamente com o monitoramento e avaliação. Para essa fase, foram destinados no acordo de empréstimo um montante de US\$ 83,45 milhões para financiar a continuidade do processo de ampliação do Saúde da Família nas grandes cidades e para o fortalecimento da capacidade avaliativa no âmbito estadual e federal. A novidade dessa segunda fase, que durou até 2015, foi a introdução de dispositivos que atrelavam os valores a serem transferidos aos estados e municípios ao alcance de metas de desenvolvimento institucional e de resultados para uma série de indicadores de saúde.

Ainda em 2008, a exemplo do que havia ocorrido em 2002, o Ministério da Saúde colocou em curso uma pesquisa amostral intitulada: 'Saúde da família no Brasil: situação atual e perspectivas', para avaliar a situação da atenção básica em todo o País. Para uma amostra de 2.133 equipes, representativa por Unidade da Federação, foram avaliados o processo de trabalho das equipes, o acesso dos usuários a serviços e procedimento, a gestão do trabalho e a infraestrutura das unidades básicas de saúde.

Com vistas ao fortalecimento das práticas avaliativas na atenção básica, outra importante iniciativa do Ministério da Saúde nesse período, em parceria com pesquisadores de várias instituições e regiões do País, além de entidades representativas de classe e da Associação Brasileira de Saúde Coletiva, foi a constituição da Rede de Pesquisa em Atenção Primária à Saúde em 2009, com o objetivo de fomentar a articulação entres pesquisadores, gestores, profissionais e usuários, além de estimular uma apropriação do conjunto da sociedade dos resultados de pesquisa para a qualificação da atenção básica.
Desde então, a rede tem se tornado um dos mais importantes espaços de reflexão, discussão e de advocacy na atenção básica. Em todos esses anos, ela se ocupou em reunir, periodicamente, pesquisadores, gestores e trabalhadores para a definição de agendas de pesquisa, análise e debate sobre os resultados de estudos realizados e, sobretudo, para a defesa política da importância da atenção básica para o fortalecimento do SUS.

Como pudemos observar, nesse breve apanhado das principais iniciativas do Ministério da Saúde no campo da avaliação da atenção básica, a primeira década dos anos 2000 apresentou-se como importante momento histórico no desenvolvimento das bases para a institucionalização da avaliação, produzindo um ambiente extremamente favorável para o que viria pela frente nos próximos anos.

\section{Consolidação da agenda: o reforço das ações de monitoramento e avaliação na atenção básica no âmbito da gestão federal (2011- 2018)}

Em 2011, apesar de o Brasil estar vivendo um momento de euforia econômica, um dos principais entraves para a implementação do SUS e, em particular, da Política Nacional de Atenção Básica estava relacionado com o seu financiamento ${ }^{\mathbf{1 7 1 8}}$. Em resposta a esse cenário, no discurso oficial do governo, havia uma intenção declarada em mitigar o subfinanciamento da atenção básica, a partir do aumento dos investimentos federais nos programas prioritários.

O modelo de financiamento vigente, contudo, que até então considerava apenas contingentes populacionais e a oferta de serviços registrados no Cadastro Nacional de Estabelecimentos de Saúde (CNES), era fortemente criticado por diversos seguimentos, inclusive pela própria Presidência 
da República, que condicionou a autorização para a ampliação do financiamento federal da atenção básica ao estabelecimento de mecanismos mais robustos e regulares de avaliação da qualidade dos serviços ofertados à população.

Para atender a essa demanda, o Ministério da Saúde instituiu, em meados de 2011, um componente de qualidade no bloco de financiamento da atenção básica, por meio da criação do Programa Nacional de Melhoria do Acesso e da Qualidade da Atenção Básica (PMAQ-AB). Pela primeira vez, o financiamento da estratégia saúde da família passou a ser determinado também pelo desempenho das equipes, aferido por meio do monitoramento de indicadores de saúde e da avaliação de padrões de qualidade. O condicionamento da transferência de recursos ao desempenho dos serviços contribuiu para um forte impulsionamento da agenda nacional de monitoramento e avaliação.

Desde o início do processo de implantação do Saúde da Família, uma das principais características da política foi o esforço permanente em manter um ritmo intenso de adesão e ampliação da cobertura populacional. Muito embora se reconhecesse os avanços e resultados atingidos pela atenção básica no Brasil, os estudos a respeito do seu funcionamento e qualidade apontavam para a necessidade do enfrentamento de problemas em diversas dimensões, desde aspectos relacionados com a infraestrutura e ambiência das unidades, passando por aspectos relacionados ao acesso, acolhimento, resolutividade e integração com os demais níveis da rede de atenção à saúde 19-21.

A cobertura populacional da saúde da família no final de 2010 já atingia mais de $50 \%$ da população brasileira, com 32.243 equipes; e o histórico de crescimento anual vinha apresentando um refreamento ao longo dos últimos anos. O desafio do financiamento e, sobretudo, a dificuldade de provimento e fixação do profissional médico nos municípios menores e mais isolados dos centros urbanos e nas periferias das grandes cidades impuseram uma expressiva diminuição nas possibilidades de expansão do Saúde da Família. Diante desse cenário, inseriu-se, de maneira mais contunde na agenda da política, a necessidade de fortalecer o desempenho dos serviços. A questão da qualidade da gestão e das práticas das equipes de atenção básica assumiu maior relevância e a preocupação com a rápida expansão foi cedendo lugar a uma maior preocupação com a qualidade e a resolutividade da atenção básica ${ }^{22,23}$.

Nesse contexto, o Ministério da Saúde colocou em curso um amplo processo de revisão da política brasileira de atenção básica em saúde que apresentou como resultado a publicação de uma nova política em outubro de 2011. No que se refere ao tema do monitoramento e avaliação, ela reforça os elementos que já haviam sido inseridos na política anterior e atrela, explicitamente, a ideia da institucionalização da avaliação a processos de qualificação da atenção básica. Figura como responsabilidade dos três níveis de gestão empreender estratégias de qualificação da atenção básica por meio do planejamento, monitoramento e avaliação das ações e serviços e, além disso, cumpre aos trabalhadores se envolver nas atividades de planejamento local, bem como o

monitoramento e avaliação das ações na sua equipe, unidade e município, visando à readequação do processo de trabalho e do planejamento diante das necessidades, realidade, dificuldades e possibilidades analisadas ${ }^{24}$.

Uma das maiores novidades inseridas na política foi o PMAQ-AB, que além de sinalizar para o aumento do financiamento federal da atenção básica, mediante o alcance de resultados, intensificou a centralidade do monitoramento e avaliação no contexto geral política. O programa, enquanto importante componente da política, passou a capitanear os esforços do Ministério da Saúde para a mobilização dos atores locais em direção 
a mudanças nas práticas de gestão, cuidado e gestão do cuidado ${ }^{22,24}$. Do ponto de vista financeiro, apenas no primeiro ano (2011) de sua execução, o programa garantiu um incremento de R\$ 70 milhões nos repasses federais para os municípios; em 2012, de aproximadamente R\$ 700 milhões; em 2013 e 2014, de aproximadamente $\mathrm{R} \$ 1,3$ bilhão; e entre 2015 e 2017, de aproximadamente $R \$ 1,7$ bilhão, sendo que, atualmente, o programa representa cerca de $10 \%$ do orçamento federal.

Entre seus principais objetivos, o PMAQ-AB pretendia provocar mudanças nas práticas de gestores e profissionais, alterando a estrutura de preferências e o comportamento desses atores. Com uma lógica fortemente vinculada ao aperfeiçoamento dos processos de gestão, o programa demarcou um conjunto de elementos que deveria ser tratado como prioridade, ganhando destaque temas como planejamento, monitoramento e avaliação, gestão do cuidado em saúde, acolhimento à demanda espontânea, dentre tantos outros que no seu conjunto pretendiam contribuir para a conformação de uma atenção básica mais acessível e resolutiva.

Em seus dois primeiros ciclos (2011 e 2013), o programa foi organizado em quatro fases complementares que deveriam conformar um ciclo contínuo de melhoria do acesso e da qualidade da atenção básica. A primeira fase, 'de adesão e contratualização', constitui um momento em que é esperada a iniciativa de gestores e trabalhadores para o engajamento nos processos de qualificação daquilo que fazem, a partir da confirmação de seu interesse em se comprometer com o desenvolvimento de uma série de medidas que pudessem qualificar a oferta de serviços aos usuários. Na segunda fase, 'de desenvolvimento', cerne e elemento estruturante do programa, os sujeitos que se comprometeram na etapa de adesão formal deveriam colocar em ação os esforços qualificadores da atenção básica, e, para isso, o programa prevê quatro estratégias mais relevantes que possam orientar o movimento dos atores: autoavaliação, monitoramento, educação permanente e apoio institucional. A terceira fase, 'de avaliação externa', constitui-se na aplicação de instrumentos avaliativos, como o nome sugere, realizada por agentes externos, cujos resultados contribuem para a composição das notas das equipes para a sua certificação. Por fim, a quarta fase, 'de recontratualização', é aquele destinada a inserir a ciclicidade no programa prevendo novo momento de contratualização, a partir dos desempenhos alcançados no ciclo anterior. No terceiro ciclo (2015), o programa passou a ser organizado em apenas três fases e um eixo estratégico transversal de desenvolvimento. A intenção dessa mudança em seu desenho foi a de desatrelar os esforços de qualificação às suas etapas procedimentais.

Uma importante inovação do programa foi articular, na etapa de avaliação externa, interesses, métodos e aplicações de modelos de avaliações normativas e pesquisas avaliativas simultaneamente. Para essa etapa, foram mobilizadas dezenas de instituições de ensino e pesquisa, que, inicialmente, foram mobilizadas no interior da Rede de Pesquisa em Atenção Primária à Saúde, sendo que muitas delas foram responsáveis pela execução de estudos avaliativos da atenção básica desenvolvidas ao longo da década anterior, em uma complexa operação de governança, formulação dos instrumentos e execução do processo de coleta de dados. No primeiro ciclo, participaram do programa e foram avaliadas 17.482 equipes (53,1\%); no segundo ciclo, 30.424 (88,7\%) e no terceiro, 38.849 (95\%).

Os instrumentos de avaliação externa contam com questões/padrões de qualidade relacionados com condições de infraestrutura e ambiência das unidades básicas de saúde, existência de equipamentos em condições de funcionamento, disponibilidade de insumos, medicamentos e imunobiológicos, a gestão do trabalho, ao acesso, acolhimento, processo de trabalho, oferta de serviços, integração com a rede de atenção à saúde e satisfação do usuário. 
Nesse período, outra dimensão que passou a fazer parte do rol de preocupações do Ministério da Saúde para a qualificação do monitoramento e avaliação da atenção básica foi o aperfeiçoamento do sistema de informação. Os dados coletados e registrados são essenciais para o processo de monitoramento e, em muitas situações, a depender do escopo e desenho, também para o processo de avaliação. Desde 1998, o Sistema de Informação da Atenção Básica (Siab) constituiu-se como a principal fonte de dados para o monitoramento e avaliação da atenção básica, e sua mais importante característica era a sua possibilidade em disponibilizar informações territorializadas para o planejamento local, seja por parte do gestor, seja por parte da equipe de saúde da família, e até mesmo do agente comunitário de saúde, na medida em que permitia a agregação de informações por microárea de atuação de cada agente ${ }^{25}$.

Foi necessário, contudo, a partir da formulação e implementação de uma estratégia de saúde eletrônica (eSaúde) para o País, implementar significativas transformações na concepção de sistema de informação para a atenção básica. O eSaúde pressupõe, entre outras coisas, a padronização de modelos de informação, a interoperabilidade entre distintos sistemas de informação nos diferentes níveis de atenção e a informação individualizada por cidadão, de forma a permitir o uso do registro eletrônico em saúde ${ }^{26}$.

Para essa nova realidade conceitual de desenvolvimento de saúde digital, o Siab apresentava uma série de limitações, inclusive do ponto de vista tecnológico, que conduziram à necessidade da constituição de um novo sistema de informação para a atenção básica. Entre as mais importantes, destacam-se o fato de que o sistema de coleta de dados era exclusivamente em formato de fichas impressas, com necessidade de posterior digitação, não permitia a informação individualizada, possuía um escopo restrito de informações para a gestão e gestão do cuidado e inviabilizava a integração com os demais sistemas que utilizam o cartão nacional de saúde para a identificação e registro das intervenções de saúde.

Em 2013, o Ministério da Saúde lançou a estratégia eSUS Atenção Básica e o Sistema de Informação em Saúde para a Atenção Básica (Sisab), com o intuito de promover avanços tecnológicos do sistema utilizado na captação de dados da atenção básica, aprimorar as ferramentas utilizadas por trabalhadores e gestores nas ações de cuidado e gestão, promover a integração e a interoperabilidade com outros sistemas de informação em saúde e buscar a reestruturação nas formas de coleta, processamento, validação e uso de informações. Com essa nova concepção para o sistema de informação na atenção básica, as possibilidades de uso dos dados coletados rotineiramente nos serviços, para iniciativas de monitoramento e avaliação, ampliaram-se significativamente. A partir de então, passou a ser possível monitorar não somente o número de atendimentos, procedimentos ou visitas domiciliares dos profissionais, mas também o nível de acesso e utilização dos serviços, na medida em que permite mensurar indicadores como o número de usuários da área de abrangência das equipes atendidos, para além do número de atendimentos, que não permite acompanhar a concentração ou dispersão do acesso entre os usuários cadastrados.

Nas situações em que as unidades básicas de saúde encontram condições para implantar o eSUS Atenção Básica na modalidade de prontuário eletrônico, o seu uso potencializa, sobremaneira, a gestão e a coordenação do cuidado, uma vez que abre a possiblidade de os profissionais da atenção básica acessarem informações mais detalhadas e em tempo oportuno, de intervenções em saúde realizadas em outros serviços e níveis de atenção nos usuários vinculados às equipes que atuam nessas unidades básicas. Para isso, os maiores desafios relacionados com a estratégia eSUS Atenção Básica têm sido: (a) a integração com demais sistemas de informação, tais como o Sistema de Monitoramento 
e Avaliação do Pré-Natal, Parto, Puerpério e Criança (Sisprenatal) e o Sistema de Informações do Programa Nacional de Imunizações (SIPNI); e (b) a implantação do prontuário eletrônico nas unidades básicas de saúde, haja vista que, das cerca de 42 mil unidades em funcionamento no Brasil, em março de 2018, apenas 19.163 utilizavam prontuário eletrônico, sendo que, dessas, 9.373 utilizavam o sistema eSUS Atenção Básica (modalidade prontuário eletrônico).

Em 2017, a Política Nacional de Atenção Básica passou por mais um momento de mudança; e, no que tange ao tema do monitoramento e avaliação, o conteúdo da política seguiu reforçando a relevância da temática enquanto responsabilidade de gestores e trabalhadores, com vistas à institucionalização e à qualificação da atenção básica, introduziu com maior detalhamento a importância de dispositivos como o da autoavaliação e reafirmou o modelo de financiamento que contempla componente de transferência de recursos condicionados ao desempenho e qualidade dos serviços ${ }^{27}$.

A exemplo do que percebemos ter ocorrido na primeira década dos anos 2000, o volume de esforços e de iniciativas do Ministério da Saúde, voltados para a consolidação das práticas de monitoramento e avaliação na atenção básica, manteve-se presente nos anos 2010, por vezes reforçando métodos, instrumentos e concepções organizativas do período anterior, e por outras, instituindo novas formas e aperfeiçoando às já existentes.

\section{Considerações finais}

A melhoria de toda e qualquer política pública passa pelo reconhecimento de seus problemas e pelo exercício de encontrar caminhos para a mitigação ou eliminação desses problemas. Contado com tal premissa, a literatura que trata da análise de políticas públicas, nos mais variados campos do conhecimento, tem dado importantes contribuições para desvelar possíveis caminhos em direção ao aperfeiçoamento das políticas. Dentre eles, tem-se destacado o papel da avaliação nesse processo.

Toda avaliação, a despeito das escolhas metodológicas do avaliador, revela apenas um recorte da realidade; e o uso de seus resultados no processo de tomada de decisão dependerá do contexto político, social e econômico no qual os diferentes atores estão inseridos. O 'bom' método confere credibilidade aos resultados dos esforços avaliativos, mas sem um engajamento e reconhecimento dos sujeitos envolvidos nas diferentes faces das políticas, muito provavelmente, não haverá mudança. No Estado liberal democrático, os incentivos institucionais para a produção de fatores que favoreçam a transparência das atividades do poder público, bem como seus efeitos, têm povoado o debate público; e, segundo nosso entendimento, sem o incentivo e investimento em medidas concretas que viabilizam a análise e compreensão do que é proposto, executado e deixado de ser executado pelo Estado, não será possível a materialização do ideal de accountability democrático tão amplamente proposto e defendido pelos teóricos da democracia.

Como descrevemos ao longo do presente ensaio, as últimas duas décadas foram particularmente profícuas na produção de experiências que viabilizaram a materialização, ao menos em parte, da agenda de institucionalização da avaliação na atenção básica. No entanto, cabe indagar quais seriam os maiores desafios a serem enfrentados na consolidação dessa agenda, sobretudo em cenários que exigem forte resiliência do sistema público de saúde e no qual se avizinham profundas restrições de ordem orçamentária e financeira, como tudo indica ser a atual situação no Brasil. Para uma incompleta e não exaustiva contribuição, entre os desafios mais relevantes para essa agenda, nos parece ganhar maior relevo: 
a. A consolidação do PMAQ-AB como principal instrumento de monitoramento, avaliação, qualificação e financiamento da atenção básica;

b. A construção e aperfeiçoamento de ferramentas e dispositivos de monitoramento e avaliação, adequados aos padrões tecnológicos atuais, capazes de contribuir para a qualificação dos processos de gestão e de gestão do cuidado;

c. O seguimento de investimentos para o aumento da capacidade avaliativa de gestores e trabalhadores da atenção básica;

d. A regulação do mercado de prontuário eletrônico na atenção básica, com a definição de critérios, funcionalidades e modelos de informação que favoreçam uma atenção básica acessível, acolhedora e resolutiva;

e. A consolidação do eSUS Atenção Básica como principal ferramenta de prontuário eletrônico na atenção básica, integrando e/ ou interoperando com os demais sistemas de informação;

f. O reforço do investimento, fomento e uso de pesquisas avaliativas para o aprimoramento da Política Nacional de Atenção Básica, e;

g. O aperfeiçoamento dos mecanismos de publicização, comunicação e uso dos resultados produzidos por processos avaliativos.

\section{Referências}

1. Silva LMV. Conceitos, abordagens e estratégias para a avaliação em saúde. In: Hartz, ZMA, Silva LMV, organizadoras. Avaliação em saúde: dos modelos teóricos à prática na avaliação de programas e sistemas de saúde. Salvador: EDUFBA; Rio de Janeiro: Fiocruz; 2005. p. 15-39.

2. Figueiró AC, Frias PG, Navarro LM. Avaliação em saúde: conceitos básicos para prática nas instituições. In: Samico I, Felisberto E, Figueiró AC, et al., organizadores. Avaliação em saúde: bases conceituais e operacionais. Rio de Janeiro: MedBook; 2010. p. 1-13.

3. Howlett M, Ramesh M, Perl A. Política pública: seus ciclos e subsistemas, uma abordagem integral. Rio de Janeiro: Elsevier; 2013.

4. Patton MQ. Utilization-focused evaluation. Londres: SAGE; 1997.

5. Faria CAP. A política da avaliação de políticas públicas. Rev. bras. Ci. Soc. [online]. 2005 out; [acesso em 2018 out 2]; 20(59): 97-110. Disponível em: http://www.scielo.br/scielo.php?pid=S0102$-092005000300007 \&$ script=sci_abstract\&tlng=pt.

6. Paim JS. Bases conceituais da reforma sanitária brasileira. In: Fleury S, organizadora. Saúde e democracia: a luta do CEBES. São Paulo: Lemos; 1997. p. 11-24. 
7. Brasil. Ministério da Saúde. Portaria no 3.925 , de 13 de novembro de 1998. Aprova o Manual para a Organização da Atenção Básica no Sistema Único de Saúde. Diário Oficial da União. 13 Nov 1998.

8. Brasil. Ministério da Saúde. Secretária de Atenção à Saúde. Avaliação na atenção básica em saúde: caminhos da institucionalização. Brasília, DF: Ministério da Saúde; 2015.

9. Brasil. Ministério da Saúde. Secretaria de Atenção à Saúde. Avaliação normativa do Programa Saúde da Família no Brasil: monitoramento da implantação e funcionamento das equipes de saúde da família: 2001-2002. Brasília, DF: Ministério da Saúde; 2004.

10. Felisberto, E. Da teoria à formulação de uma política nacional de avaliação em saúde: reabrindo o debate. Ciênc Saúde Colet. [internet]. 2006 set [acesso em 2018 out 2]; 11(3):553563. Disponível em: http://www.scielo.br/scielo. php?pid=S1413-81232006000300002\&script=sci abstract\&tlng=es.

11. Felisberto E, Bezerra LCA, Albuquerque AC. Avaliação na estratégia saúde da família: os sentidos da institucionalização dessa prática. In: Sousa MF, Franco MS, Mendonça AVM, organizadores. Saúde da família nos municípios brasileiros: os reflexos dos 20 anos no espelho do futuro. Campinas: Saberes; 2014. p. 811-843.

12. Bodstein R, Feliciano K, Hortale VA, et al. Estudos de linha de base do projeto de expansão e consolidação do saúde da família (ELB/Proesf): considerações sobre seu acompanhamento. Ciênc Saúde Colet. [internet]. 2006 set [acesso em 2018 out 2]; 11(3):725-731. Disponível em: http://www.scielo.br/ pdf/csc/v1ln3/30986.pdf.

13. Almeida PF, Giovanella L. Avaliação em Atenção Básica à Saúde no Brasil: mapeamento e análise das pesquisas realizadas e/ou financiadas pelo Ministério da Saúde entre os anos de 2000 e 2006. Cad Saúde Pública. [internet]. 2008 ago [acesso em 2018 out 2]; 24(8):1727-1742. Disponível em: http://www. scielo.br/scielo.php?script=sci_arttext\&pid=S0102-
$-311 X 2008000800002$.

14. Natal S, Santos EM, Alves CKA, et al. A implementação do projeto estratégico de fortalecimento da capacidade técnica em monitoramento e avaliação das secretarias de saúde: avaliação formativa e auto-avaliação. In: Hartz ZMA, Felisberto E, Silva LMV, organizadores. Meta-avaliação da atenção básica à saúde: teoria e prática. Rio de Janeiro: Fiocruz; 2008. p. 341-367.

15. Brasil. Ministério da Saúde. Secretaria de Atenção à Saúde. Departamento de Atenção Básica. Caderno de Auto-Avaliação. Brasília, DF: Ministério da Saúde; 2009.

16. Brasil. Ministério da Saúde. Portaria ${ }^{\circ}$ 648, de 28 de março de 2006. Dispõe sobre a revisão de diretrizes e normas para a organização da Atenção Básica para o Programa da Saúde da Família (PSF) e o Programa de Agentes Comunitários de Saúde (PACS). Diário Oficial da União. 29 Mar 2006.

17. BRASIL. Conselho Nacional de Secretarias Municipais de Saúde. A atenção básica que queremos. Brasília, DF: Conasems; 2011. [acesso em 2018 out 2]. Disponível em: https:// www.paho.org/bra/index.php?option=com docman\&view=download\&category_slug=servicos- aude-095\&alias=1396-a-atencao-basica-que-queremos- $6 \&$ Itemid $=965$.

18. Mendes A, Marques RM. A dimensão do financiamento da atenção básica e da estratégia saúde da família no contexto do SUS. In: Sousa MF, Franco MS, Mendonça AVM, organizadores. Saúde da família nos municípios brasileiros: os reflexos dos 20 anos no espelho do futuro. Campinas: Saberes; 2014. p. 458-517.

19. Giovanella L, Mendonça MHM, Almeida PF, et al. Saúde da família: limites e possibilidades para uma abordagem integral de atenção primária à saúde no Brasil. Ciênc Saúde Colet. [internet]. 2009 jun; [acesso em 2018 out 2]; 14(3):783-794. Disponível em: http://www.scielo.br/scielo.php?pid=S1413$-1232009000300014 \&$ script=sci_abstract $\&$ tlng=es. 
20. Facchini LA, Piccini RX, Tomasi E, et al. Desempenho do PSF no sul e no nordeste do Brasil: avaliação institucional e epidemiológica da atenção básica à saúde. Ciênc Saúde Colet. [internet]. 2006 set [acesso em 2018 out 2]; 11(3):669-681. Disponível em: http://www.scielo.br/scielo.php?pid=S1413$-1232006000300015 \&$ script=sci_abstract $\&$ tlng=pt.

21. Medina MG, Hartz ZMA. The role of the Family Health Program in the organization of primary care in municipal health systems. Cad Saúde Pública. [internet]. 2009 maio [acesso em 2018 out 2] 25(5):1153-1167. Disponível em: http://www.scielo. br/pdf/csp/v25n5/22.pdf.

22. Pinto HA, Sousa A, Florêncio AR. O programa nacional de melhoria do acesso e da qualidade da atenção básica: reflexões sobre o seu desenho e processo de implantação. RECIIS. [internet]. 2012 ago [acesso em 2018 out 2]; 6(2). 1-19. Disponível em: https://www.reciis.icict.fiocruz.br/index.php/reciis/ article/view/492/1142.

23. Pinto HA, Sousa ANA, Ferla AA. O programa nacional de melhoria do acesso e da qualidade da atenção básica: várias faces de uma política inovadora. Saúde em debate. [internet]. 2014 out [acesso em 2018 out 2]; 38(esp):358-372. Disponível em: http://www. scielo.br/scielo.php?script=sci_arttext_pr\&pid=SO1 031042014000600358\&tlng=pt.
24. Brasil. Ministério da Saúde. Portaria no 2.488, de 21 de outubro de 2011. Aprova a Política Nacional de Atenção Básica, estabelecendo a revisão de diretrizes e normas para a organização da Atenção Básica, para a Estratégia Saúde da Família (ESF) e o Programa de Agentes Comunitários de Saúde (PACS). Diário Oficial da União. 22 Out 2011.

25. Aquino R, Medina MG, Abdon C. Monitoramento e avaliação: reflexos visíveis na atenção primária à saúde no Brasil. In: Sousa MF, Franco MS, Mendonça AVM, organizadores. Saúde da família nos municípios brasileiros: os reflexos dos 20 anos no espelho do futuro. Campinas: Saberes; 2014. p. 893-932.

26. Brasil. Ministério da Saúde. Estratégia eSaúde para o Brasil. Brasília, DF: Ministério da Saúde; 2017.

27. Brasil. Ministério da Saúde. Portaria n ${ }^{0}$ 2.436, de 21 de setembro de 2017. Aprova a Política Nacional de Atenção Básica, estabelecendo a revisão de diretrizes para a organização da Atenção Básica, no âmbito do Sistema Único de Saúde (SUS). Diário Oficial da União. 22 Set 2017.

Recebido em 12/06/2018

Aprovado em 05/10/2018

Conflito de interesses: inexistente

Suporte financeiro: não houve 